\title{
Development of Ferrocene-Based Planar Chiral Imidazopyridinium Salts for Catalysis
}

\author{
Keegan P. Fitzpatrick, C. Benjamin Schwamb, Christopher T. Check, Ki-Po Jang, David N. \\ Barsoum, and Karl A. Scheidt \\ Department of Chemistry, Center for Molecular Innovation and Drug Discovery, Chemistry of \\ Life Processes Institute, Northwestern University, Evanston, IL 60208 (USA)

\section{Supporting Information}

\section{Table of Contents}

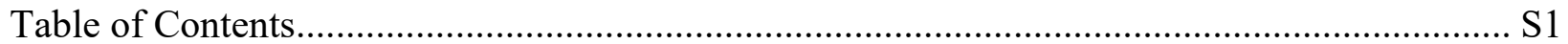

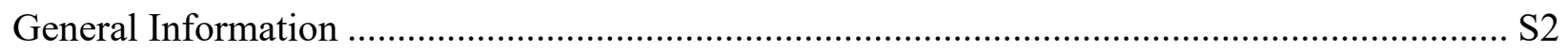

General Synthetic Procedures and Spectral Data for New Compounds.................................. S3

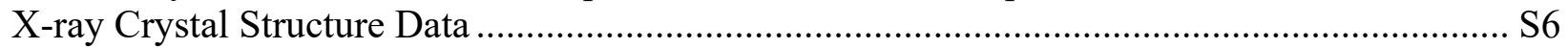

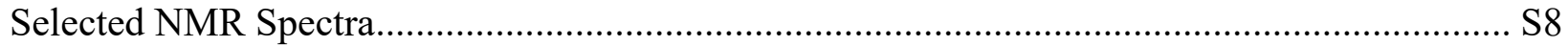

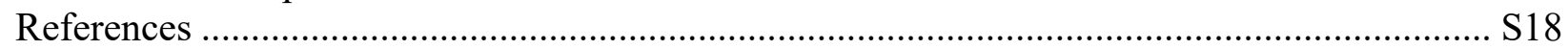




\section{General Information}

All reactions were carried out under a nitrogen atmosphere in oven-dried glassware with magnetic stirring. THF was purified by passage through a bed of activated alumina. Reagents were purified prior to use unless otherwise stated following the guidelines of Perrin and Armarego. ${ }^{1}$ Silicycle SiliaFlash P60 silica gel 60 (230-400 mesh) was used for column chromatography. Analytical thin layer chromatography was performed on EM Reagent $0.25 \mathrm{~mm}$ silica gel $60-\mathrm{F}$ plates. Visualization was accomplished with UV light. ${ }^{1} \mathrm{H}$ NMR spectra were recorded on AVANCE III $500 \mathrm{MHz}$ w/ direct cryoprobe $(500 \mathrm{MHz})$ spectrometer and are reported in ppm using solvent as an internal standard $\left(\mathrm{CDCl}_{3}\right.$ at $7.26 \mathrm{ppm})$. Data are reported as ( ap = apparent, $\mathrm{s}=$ singlet, $\mathrm{d}=$ doublet, $\mathrm{t}=$ apparent triplet, $\mathrm{q}=$ quartet, $\mathrm{m}=$ multiplet, $\mathrm{b}=$ broad; coupling constant(s) in $\mathrm{Hz}$; integration.) Proton-decoupled ${ }^{13} \mathrm{C}$ NMR spectra were recorded on an AVANCE III $500 \mathrm{MHz}$ w/ direct cryoprobe $(125 \mathrm{MHz})$ spectrometer and are reported in ppm using solvent as an internal standard $\left(\mathrm{CDCl}_{3}\right.$ at $\left.77.16 \mathrm{ppm}\right) .{ }^{77} \mathrm{Se}$ spectra were acquired at $26^{\circ} \mathrm{C}$ on a $400 \mathrm{MHz}$ Agilent 400MR-DD2 spectrometer equipped with a OneNMR probe and a 7600AS autosampler. Mass spectra were obtained on a WATERS Acquity-H UPLC-MS with a single quad detector (ESI) or an Agilent 7890 gas chromatograph equipped with a 5975C single quadrupole EI-MS. Compounds 8a-d, 13a, 14a-b, 15a-b, 16a, and 20a-d have all been synthesized and fully characterized by our lab. ${ }^{2,3}$ X-ray structures for (+)-14a, (-)-15a, (+)-15b, (-)-16a, (-)-20a, (-)-20b, (-)-20c, and (-)-20d have all been submitted to the CCDC and assigned the following numbers: (+)-14a (1007913), (-)-15a (1007914), (+)15b (1875415), (-)16a (1875413), (-)-20a (1016510), (-)-20b (1016507), (-)-20c (1016508), (-)-20d (1016509). Buried volume calculations were performed using the SambVca 2 web application available online at https://www.molnac.unisa.it/OMtools/sambvca2.0/index.html. The crystal structures of $\mathrm{CuCl}$ complexes 15a-b and 16a were uploaded in .xyz format. The xz plane was defined by the imidazopyridine ring system, the z-axis was oriented along the xz projection of NHC-metal bond, and the center of the sphere was set at a distance of $2.0 \AA$ distance from the carbene carbon along the $\mathrm{z}$-axis. All $\mathrm{Cu}, \mathrm{Cl}$, and hydrogen atoms were excluded from the analysis. All calculations used atomic radii as described by Bondi scaled by a factor of 1.17 . For the sake of clarity and comparison, the coordinates from (-)-15a and (-)-16a were inverted in Figure 5. Buried volume is not dependent on handedness as it measures the interaction of a hypothetical ligand with an isotropic sphere.

Standardization of heteronuclear spectra was conducted using the unified scale according to eqn. (1) as described in Pure Appl. Chem. 2008, 80, 59-84.

$$
\text { (1) } \Xi_{X}=100 \times\left(v_{X} / v_{o}\right)
$$

where $v_{o}=$ the absolute ${ }^{1} \mathrm{H}$ frequency of $0.00 \mathrm{ppm}\left(\delta_{\mathrm{TMS}}\right)$ for experiments conducted in $\mathrm{CDCl}_{3}$; $\mathrm{OR} v_{o}=$ $\delta_{\mathrm{TMS}}+0.062 \mathrm{ppm}$ for DMSO, OR $\delta_{\mathrm{TMS}}-0.160$ for acetone- $d_{6}$, taken consecutively with heteronucelar experiments.

$\Xi_{77 S e}=19.071513$

and $v_{x}=$ abosolute frequency of the 0 ppm position for isotope $x$. 


\title{
General Synthetic Procedures and Spectral Data for New Compounds
}

\author{
General Procedure A for the synthesis of planar chiral imidazolium salts 8 :
}

To an oven-dried vial with a stir bar under $\mathrm{N}_{2}$ atm, aldehyde (1.0 equiv.) and amine or aniline derivative (1.0 equiv.) were dissolved in THF $(0.1 \mathrm{M})$. To another oven-dried vial under $\mathrm{N}_{2} \mathrm{~atm}$, was added paraformaldehyde (1.2 equiv.) and anhydrous $\mathrm{ZnCl}_{2}$ (1.2 equiv.). Note: the $\mathrm{ZnCl}_{2}$ should be kept in a glovebox. If it looks wet, or has been open to air, the reaction will not work. To this mixture of solids was added concentrated $\mathrm{HCl}$ (2.4 equiv.) which was then added dropwise to the solution of aldehyde, amine, and THF. The reaction slowly turned dark orange/red and was allowed to stir for $1 \mathrm{~h}$ or until completed by TLC (DCM:MeOH:AcOH, 90:10:1). The reaction was concentrated and dissolved in the minimum amount of DCM. The crude mixture was purified by column chromatography on neutral alumina. The impurities were eluted with DCM, and the product eluted as an orange band ( $3 \% \mathrm{EtOH}$ in DCM). If needed, the resulting orange solid was washed with acetone to purify further.

General Procedure B for the synthesis of selenourea compounds 21:

A modified synthesis of a reported procedure was used. ${ }^{4}$ The NHC salt $(0.04 \mathrm{mmol})$ and $\mathrm{KO}^{t} \mathrm{Bu}(1.2-1.8$ equiv.) were weighed into an oven-dried vial. Excess Se $(10 \mathrm{mg})$ was added at $-76^{\circ} \mathrm{C}$, followed by a stir bar, and the vial was purged with $\mathrm{N}_{2}$. Dry THF $(1 \mathrm{~mL})$ was added and the resulting suspension was stirred at room temperature overnight. The solvent was then removed under vacuum, diluted with DCM (5 mL), and passed through a pad of celite. The celite was washed with more DCM $(3 \times 5 \mathrm{~mL})$ and dried under vacuum. The crude product was loaded on a short alumina column and eluted with DCM to afford the pure product.
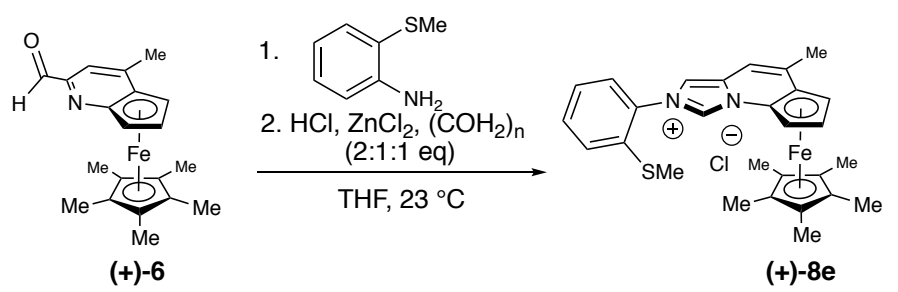

$(+)$-Ferrocenyl 2-methylthiophenyl imidazolium chloride, $(+)-8 e$ : Prepared according to the general procedure A using aldehyde (+)-6 (200 mg), 2-(methylthio)aniline (80 mg), $\mathrm{ZnCl}_{2}(94 \mathrm{mg})$, paraformaldehyde $(21 \mathrm{mg}), \mathrm{HCl}(0.11 \mathrm{~mL})$ and THF $(6 \mathrm{~mL})$ to afford $(+)-8 \mathrm{e}$ as a dark red solid $(185 \mathrm{mg}$, $62 \%$ yield). Analytical data for (+)-8e. ${ }^{1} \mathrm{H}$ NMR $(500 \mathrm{MHz}, \mathrm{Chloroform}-d) \delta 12.29(\mathrm{~s}, 1 \mathrm{H}), 7.68(\mathrm{~s}, 1 \mathrm{H})$, $7.57-7.48(\mathrm{~m}, 2 \mathrm{H}), 7.47-7.36(\mathrm{~m}, 2 \mathrm{H}), 6.83(\mathrm{~s}, 1 \mathrm{H}), 6.22(\mathrm{~s}, 1 \mathrm{H}), 4.20-4.10(\mathrm{~m}, 1 \mathrm{H}), 4.03-3.95(\mathrm{~m}$, $1 \mathrm{H}), 2.51(\mathrm{~s}, 3 \mathrm{H}), 2.36(\mathrm{~s}, 3 \mathrm{H}), 1.66(\mathrm{~s}, 15 \mathrm{H}) .{ }^{13} \mathrm{C} \mathrm{NMR}\left(126 \mathrm{MHz}, \mathrm{CDCl}_{3}\right) \delta 141.0,134.6,133.3,131.5$, 130.0, 128.2, 127.9, 127.3, 114.8, 105.3, 89.5, 81.5, 77.4, 73.6, 65.9, 65.1, 19.5, 16.6, 9.9. LCMS (ESI): Mass calcd for $\mathrm{C}_{24} \mathrm{H}_{31} \mathrm{FeN}_{2} \mathrm{~S}[\mathrm{M}]^{+}$, 483.4; found 483.1.

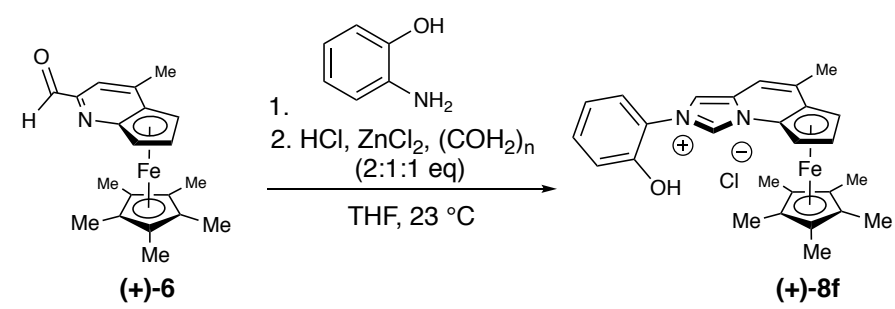

$(+)$-Ferrocenyl 2-phenol imidazolium chloride, $(+)-8 f$ : Prepared according to the general procedure A using aldehyde $(+)-6(100 \mathrm{mg})$, 2-aminophenol $(31 \mathrm{mg}), \mathrm{ZnCl}_{2}(47 \mathrm{mg})$, paraformaldehyde $(10.3 \mathrm{mg})$, $\mathrm{HCl}(55 \mu \mathrm{L})$ and THF $(3.0 \mathrm{~mL})$ to afford $(+)-8 f$ as a dark red solid $(260 \mathrm{mg}$, 53\% yield). Analytical data 
for (+)-8f. ${ }^{1} \mathrm{H}$ NMR $(500 \mathrm{MHz}$, Chloroform- $d$ ) $\delta 11.19(\mathrm{~s}, 1 \mathrm{H}), 7.68(\mathrm{~s}, 1 \mathrm{H}), 7.27(\mathrm{~s}, 1 \mathrm{H}), 6.97$ - $6.74(\mathrm{~m}$, $3 \mathrm{H}), 6.31(\mathrm{t}, J=7.3 \mathrm{~Hz}, 1 \mathrm{H}), 4.94(\mathrm{~s}, 1 \mathrm{H}), 4.12(\mathrm{t}, J=2.9 \mathrm{~Hz}, 1 \mathrm{H}), 3.92(\mathrm{t}, J=2.7 \mathrm{~Hz}, 1 \mathrm{H}), 2.32(\mathrm{~s}, 3 \mathrm{H})$, $1.58(\mathrm{~d}, J=2.7 \mathrm{~Hz}, 15 \mathrm{H}) .{ }^{13} \mathrm{C}$ NMR $\left(126 \mathrm{MHz}, \mathrm{CDCl}_{3}\right) \delta 138.5,130.3,128.8,127.3,123.7,123.2,121.4$, 112.1, 106.4, 89.4, 81.4, 72.5, 65.6, 62.0, 19.4, 9.7. LCMS (ESI): Mass calcd for $\mathrm{C}_{27} \mathrm{H}_{29} \mathrm{FeN}_{2} \mathrm{O}[\mathrm{M}]^{+}$, 453.3; found 453.3.

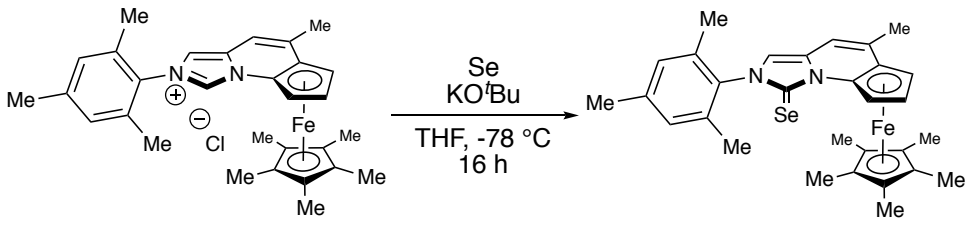

$(+)-8 \mathbf{a}$

$(+)-21 a$

$(+)$-Ferrocenyl mesityl selenourea, (+)-21a: Prepared according to the general procedure B using imidazolium chloride $(+)$-8a to afford $(+)-21$ a as an orange solid $(15.3 \mathrm{mg}, 65 \%)$. Analytical data for $(+)-$ 21a: ${ }^{1} \mathrm{H}$ NMR $(400 \mathrm{MHz}$, Acetone- $d 6) \delta 7.32(\mathrm{~s}, 1 \mathrm{H}), 7.01(\mathrm{~d}, \mathrm{~J}=10.9 \mathrm{~Hz}, 2 \mathrm{H}), 6.76(\mathrm{~s}, 1 \mathrm{H}), 6.63(\mathrm{dd}, \mathrm{J}$ $=1.1,1.0 \mathrm{~Hz}, 1 \mathrm{H}), 4.06(\mathrm{dd}, \mathrm{J}=1.1,1.0 \mathrm{~Hz}, 1 \mathrm{H}), 3.85$, (t, J = 2.1 Hz, 1H), $2.33(\mathrm{~s}, 3 \mathrm{H}), 2.23(\mathrm{~s}, 3 \mathrm{H}), 2.10$ $(\mathrm{s}, 3 \mathrm{H}), 1.92(\mathrm{~s}, 3 \mathrm{H}), 1.74(\mathrm{~s}, 15 \mathrm{H}) ;{ }^{13} \mathrm{C}$ NMR $(125 \mathrm{MHz}$, Acetone-d6) $\delta$ 150.9, 139.5, 136.7, 136.2, 135.8, 135.2, 130.9, 129.7, 129.6, 113.8, 108.9, 94.6, 81.2, 77.5, 71.3, 65.3, 64.9, 21.1, 18.9, 18.7, 18.3, $10.4 ;{ }^{77} \mathrm{Se}$ NMR (76 MHz, Acetone-d6) $\delta$ 94.47.

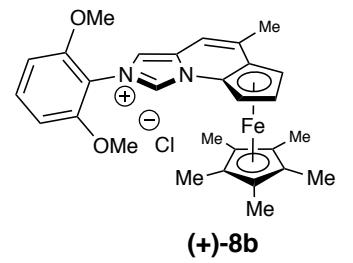

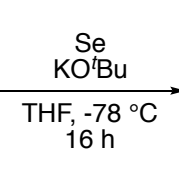

$(+)-8 b$

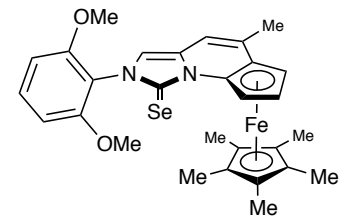

$(+)-21 b$

$(+)$-Ferrocenyl 2,6-dimethoxyphenyl selenourea, $(+)-21 \mathrm{~b}$ : Prepared according to the general procedure $\mathrm{B}$ using imidazolium chloride $(+)-8 \mathbf{b}$ to afford $(+)-\mathbf{2 1 b}$ as an orange solid $(21.0 \mathrm{mg}, 87 \%)$. Analytical data for (+)-21b: ${ }^{1} \mathrm{H}$ NMR $(500 \mathrm{MHz}$, Acetone- $d 6) \delta 7.43(\mathrm{t}, \mathrm{J}=8.5 \mathrm{~Hz}, 1 \mathrm{H}), 7.22(\mathrm{~s}, 1 \mathrm{H}), 6.80,(\mathrm{t}, \mathrm{J}=7.5 \mathrm{~Hz}$, $2 \mathrm{H}), 6.71(\mathrm{~d}, \mathrm{~J}=1.6 \mathrm{~Hz}, 1 \mathrm{H}), 6.64(\mathrm{dd}, \mathrm{J}=1.3,1.3 \mathrm{~Hz}, 1 \mathrm{H}), 4.00(\mathrm{dd}, \mathrm{J}=1.4,1.3 \mathrm{~Hz}, 1 \mathrm{H}), 3.80(\mathrm{t}, \mathrm{J}=2.6$ $\mathrm{Hz}, 1 \mathrm{H}), 3.79(\mathrm{~s}, 3 \mathrm{H}), 3.75(\mathrm{~s}, 3 \mathrm{H}), 2.21(\mathrm{~d}, \mathrm{~J}=1.0 \mathrm{~Hz}, 3 \mathrm{H}), 1.73(\mathrm{~s}, 15 \mathrm{H}) ;{ }^{13} \mathrm{C}$ NMR $(125 \mathrm{MHz}$, Acetoned6) $\delta 157.6,157.5,153.1,134.1,131.3,130.0,117.9,115.6,108.9,105.6,94.4,81.3,77.5,71.1,65.1$, $56.5,56.4,18.9,10.2 ;{ }^{77} \mathrm{Se}$ NMR (76 MHz, Acetone- $\left.d 6\right) \delta 99.96$.

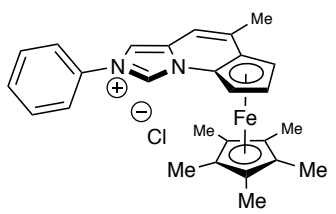

$(+)-8 c$

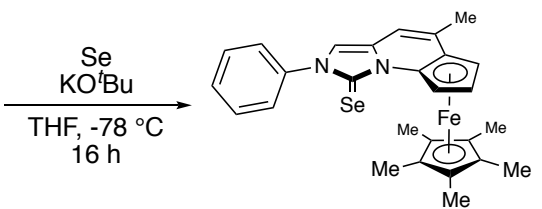

$(+)-21 c$

$(+)$-Ferrocenyl phenyl selenourea, $(+)-21 c$ : Prepared according to the general procedure B using imidazolium chloride $(+)-8 c$ to afford $(+)-21 \mathrm{c}$ as an orange solid $(20.7 \mathrm{mg}, 95 \%)$. Analytical data for $(+)-$ 21c: ${ }^{1} \mathrm{H}$ NMR $(500 \mathrm{MHz}$, Acetone- $d 6) \delta$ 7.65-7.62 (m, 2H), 7.57-7.48 (m, 4H), $6.76(\mathrm{~d}, \mathrm{~J}=1.8 \mathrm{~Hz}, 1 \mathrm{H})$, $6.67(\mathrm{dd}, \mathrm{J}=1.8,1.7 \mathrm{~Hz}, 1 \mathrm{H}), 4.05$ (dd, J = 1.8, $1.7 \mathrm{~Hz}, 1 \mathrm{H}), 3.85$ (t, J = 3.4 Hz, 1H), 2.23 (d, J = 1.7 Hz, $3 \mathrm{H}), 1.71(\mathrm{~s}, 15 \mathrm{H}) ;{ }^{13} \mathrm{C}$ NMR $(125 \mathrm{MHz}$, Acetone- $d 6) \delta$ 151.6, 140.4, 135.4, 130.6, 129.7, 129.2, 128.0, $114.9,108.6,94.1,81.3,77.7,71.5,65.6,65.5,18.9,10.1 ;{ }^{77}$ Se NMR (76 MHz, Acetone-d6) $\delta 111.89$. 

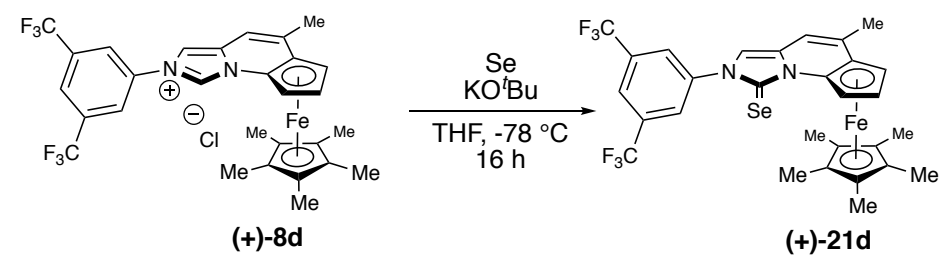

(+)-Ferrocenyl 3,5-bis(trifluoromethyl)phenyl selenourea, $(+)-21 \mathrm{~d}$ : Prepared according to the general procedure B using imidazolium chloride $(+)-8 d$ to afford $(+)-21 d$ as an orange solid $(24.8 \mathrm{mg}, 91 \%)$. Analytical data for (+)-21d: ${ }^{1} \mathrm{H}$ NMR $(500 \mathrm{MHz}$, Acetone- $d 6) \delta 8.50(\mathrm{~s}, 2 \mathrm{H}), 8.18(\mathrm{~s}, 1 \mathrm{H}), 7.86(\mathrm{~s}, 1 \mathrm{H})$, $6.79(\mathrm{~d}, \mathrm{~J}=1.4 \mathrm{~Hz}, 1 \mathrm{H}), 6.57(\mathrm{dd}, \mathrm{J}=1.4,1.2 \mathrm{~Hz}, 1 \mathrm{H}), 2.24(\mathrm{~d}, \mathrm{~J}=1.4 \mathrm{~Hz}, 3 \mathrm{H}), 1.70(\mathrm{~s}, 15 \mathrm{H}) ;{ }^{13} \mathrm{C}$ NMR $(125 \mathrm{MHz}$, Acetone- $d 6) \delta 151.9,141.6,136.5,132.8,132.5,132.3,131.9,131.2,129.0,127.3,125.2$, 123.0, 122.8, 120.8, 114.5, 108.4, 93.7, 81.5, 77.6, 71.8, 65.8, 65.7, 18.9, 10.2; ${ }^{77} \mathrm{Se}$ NMR (76 MHz, Acetone-d6) $\delta 117.36$. 


\section{X-ray Crystal Structure Data}

Determination of Structure of $(+)$-Ferrocenyl mesityl selenourea, $(+)-21$.

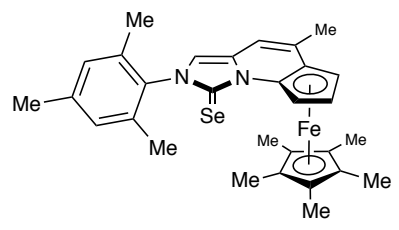

$(+)-21 a$

Single crystals of $\mathrm{C}_{30} \mathrm{H}_{34} \mathrm{FeN}_{2} \mathrm{Se}(+)-21$ a were recrystallized via the slow evaporation of acetone at $23{ }^{\circ} \mathrm{C}$. A suitable crystal was selected and mounted in inert oil and transferred to the cold gas stream of 'Bruker APEX-II COD' diffractometer. The crystal was kept at $100.02 \mathrm{~K}$ during data collection. Using Olex $2^{5}$, the structure was solved with the ShelXT ${ }^{6}$ structure solution program using Intrinsic Phasing and refined with the ShelXL ${ }^{7}$ refinement package using Least Squares minimisation. SADABS-2014/5 (Bruker, 2014/5) was used for absorption correction.

Crystal Data for $\mathrm{C}_{30} \mathrm{H}_{34} \mathrm{FeN}_{2} \mathrm{Se}(M=557.40 \mathrm{~g} / \mathrm{mol})$ : monoclinic, space group P2 $1 / \mathrm{n}$ (no. 14), $a=16.5941(8) \AA, b=$ 8.5541(4) $\AA, c=18.8413(9) \AA, \beta=106.008(3)^{\circ}, V=2570.8(2) \AA^{3}, Z=4, T=100.02 \mathrm{~K}, \mu(\mathrm{CuK} \alpha)=6.467 \mathrm{~mm}^{-}$ ${ }^{1}$, Dcalc $=1.440 \mathrm{~g} / \mathrm{cm}^{3}, 29416$ reflections measured $\left(6.294^{\circ} \leq 2 \Theta \leq 133.244^{\circ}\right), 4533$ unique $\left(R_{\text {int }}=0.0565, R_{\text {sigma }}=\right.$ 0.0384 ) which were used in all calculations. The final $R_{1}$ was 0.0294 (I $\left.>2 \sigma(\mathrm{I})\right)$ and $w R_{2}$ was 0.0750 (all data). Further information can be found in the CIF file. This crystal structure was deposited in the Cambridge Crystallographic Data Centre and assigned as 1999567.

Refinement Details: The enhanced rigid-bond restraint (SHELX keyword RIGU) was applied on the disordered Cp* ring.

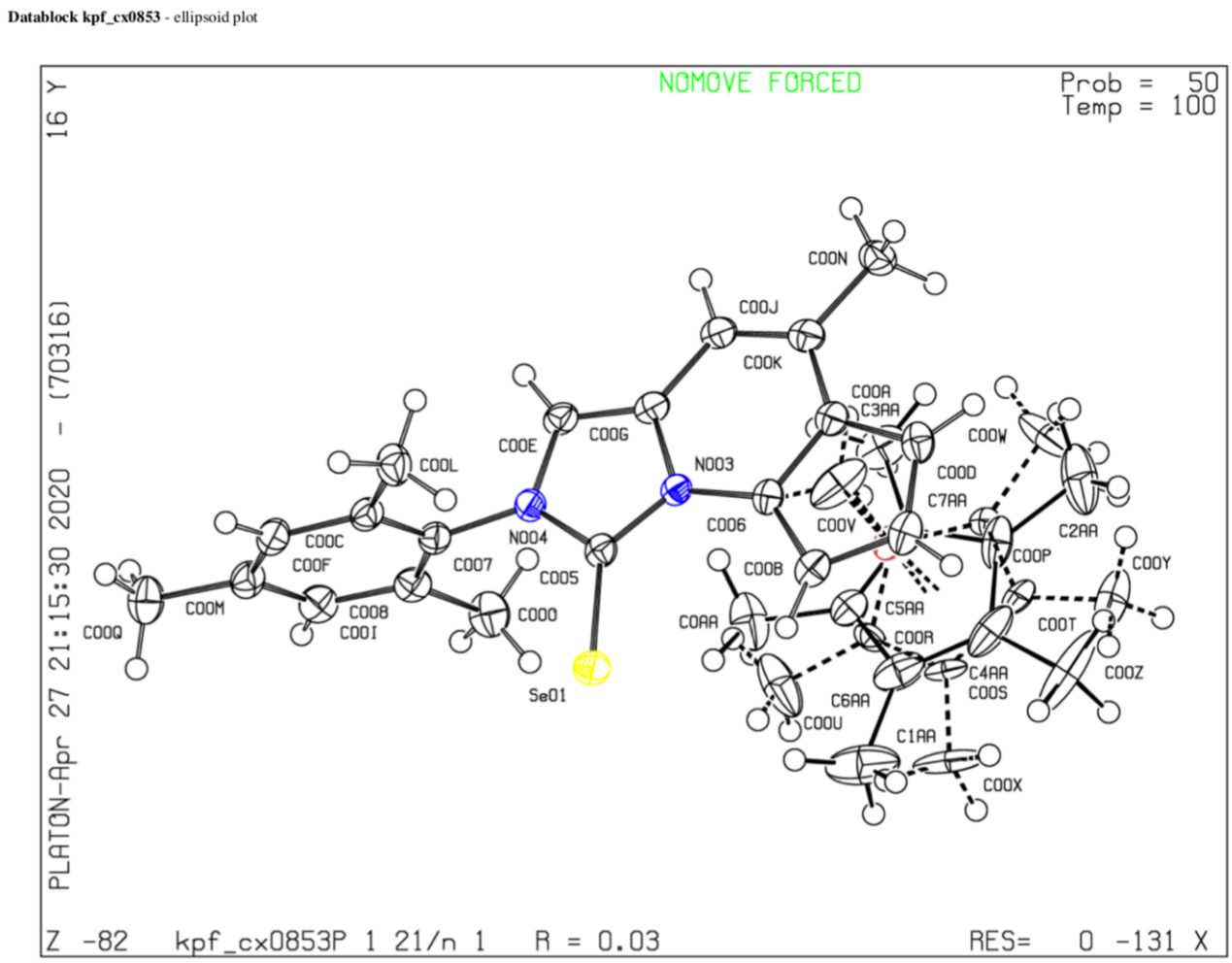

Figure S1. Structure of (+)-Ferrocenyl mesityl selenourea, $(+)-21$. 
Determination of Structure of (+)-Ferrocenyl phenyl selenourea, $(+)-21 \mathrm{c}$.

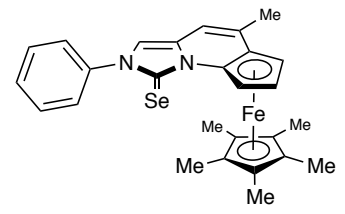

(+)-21c

Single crystals of $\mathrm{C}_{27} \mathrm{H}_{28} \mathrm{FeN} \mathrm{Fe}_{2} \mathrm{Se}(+)-21 \mathrm{c}$ were recrystallized via the slow evaporation of acetone at $23{ }^{\circ} \mathrm{C}$. A suitable crystal was selected and mounted in inert oil and transferred to the cold gas stream of 'Bruker APEX-II COD' diffractometer. The crystal was kept at $100.02 \mathrm{~K}$ during data collection. Using Olex $2^{5}$, the structure was solved with the ShelXT ${ }^{6}$ structure solution program using Intrinsic Phasing and refined with the ShelXL ${ }^{7}$ refinement package using Least Squares minimisation.

Crystal Data for $\mathrm{C}_{27} \mathrm{H}_{28} \mathrm{FeN} \mathrm{N}_{2} \mathrm{Se} \quad(M=515.32 \mathrm{~g} / \mathrm{mol})$ : orthorhombic, space group Pbca (no. 61), $a=$ 13.5714(6) $\AA, b=8.4756(4) \AA, c=40.6140(19) \AA, V=4671.7(4) \AA^{3}, Z=8, T=100.0 \mathrm{~K}, \mu(\mathrm{CuK \alpha})=7.071 \mathrm{~mm}^{-}$ ${ }^{1}$, Dcalc $=1.465 \mathrm{~g} / \mathrm{cm}^{3}, 19697$ reflections measured $\left(7.834^{\circ} \leq 2 \Theta \leq 133.386^{\circ}\right), 4098$ unique $\left(R_{\text {int }}=0.0682, \mathrm{R}_{\text {sigma }}=\right.$ 0.0540 ) which were used in all calculations. The final $R_{1}$ was 0.0519 (I $>2 \sigma(\mathrm{I})$ ) and $w R_{2}$ was 0.1243 (all data). Further information can be found in the CIF file. This crystal structure was deposited in the Cambridge Crystallographic Data Centre and assigned as 1999565.

Refinement Details: No special refinement necessary.

Datablock kpf_cx0857 - ellipsoid plot

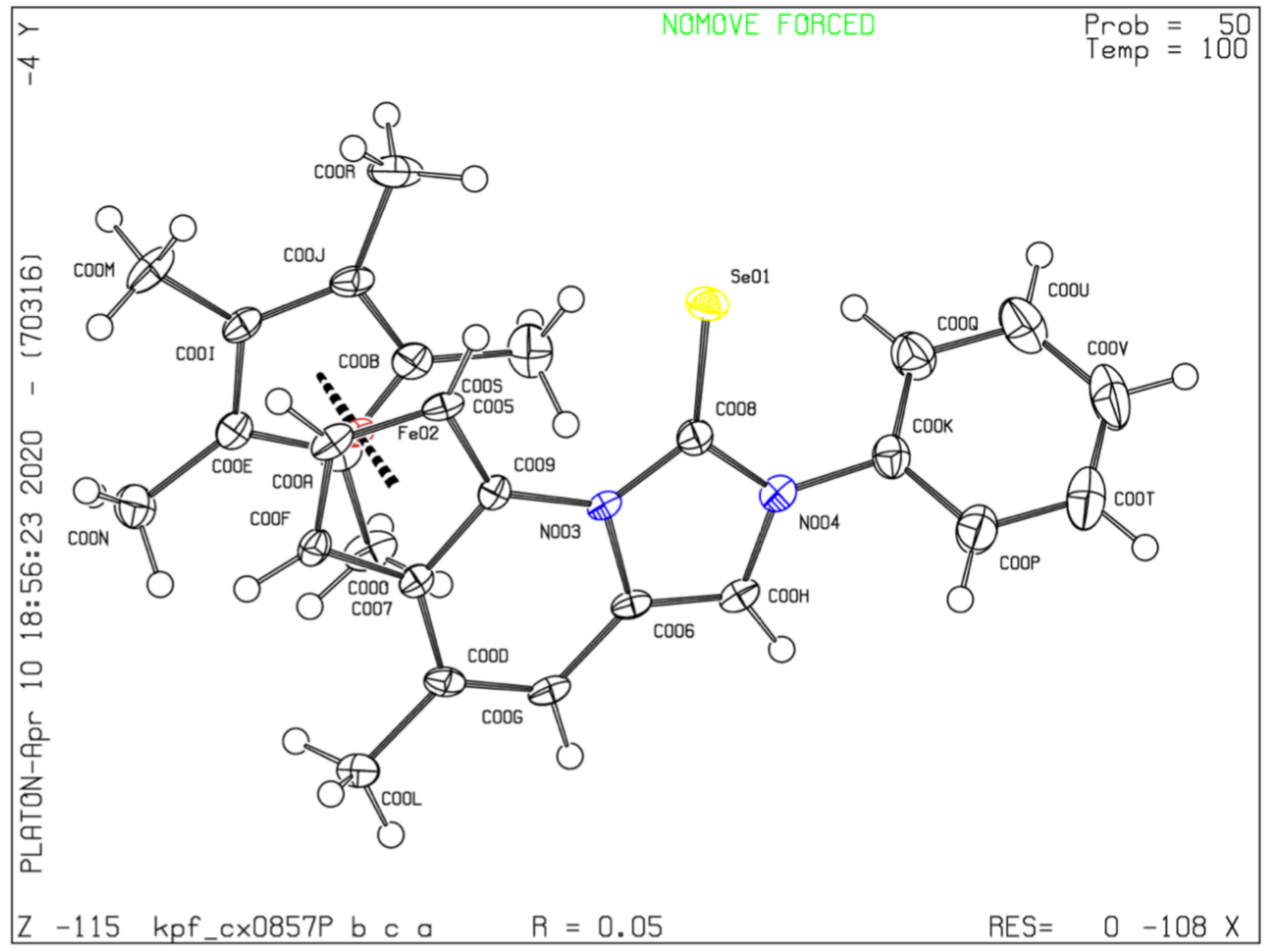

Figure S2. Structure of (+)-Ferrocenyl phenyl selenourea, $(+)-21 c$. 


\section{Selected NMR Spectra}

Figure S3. ${ }^{1} \mathrm{HNMR}$ of $(+)-8 \mathrm{e}$

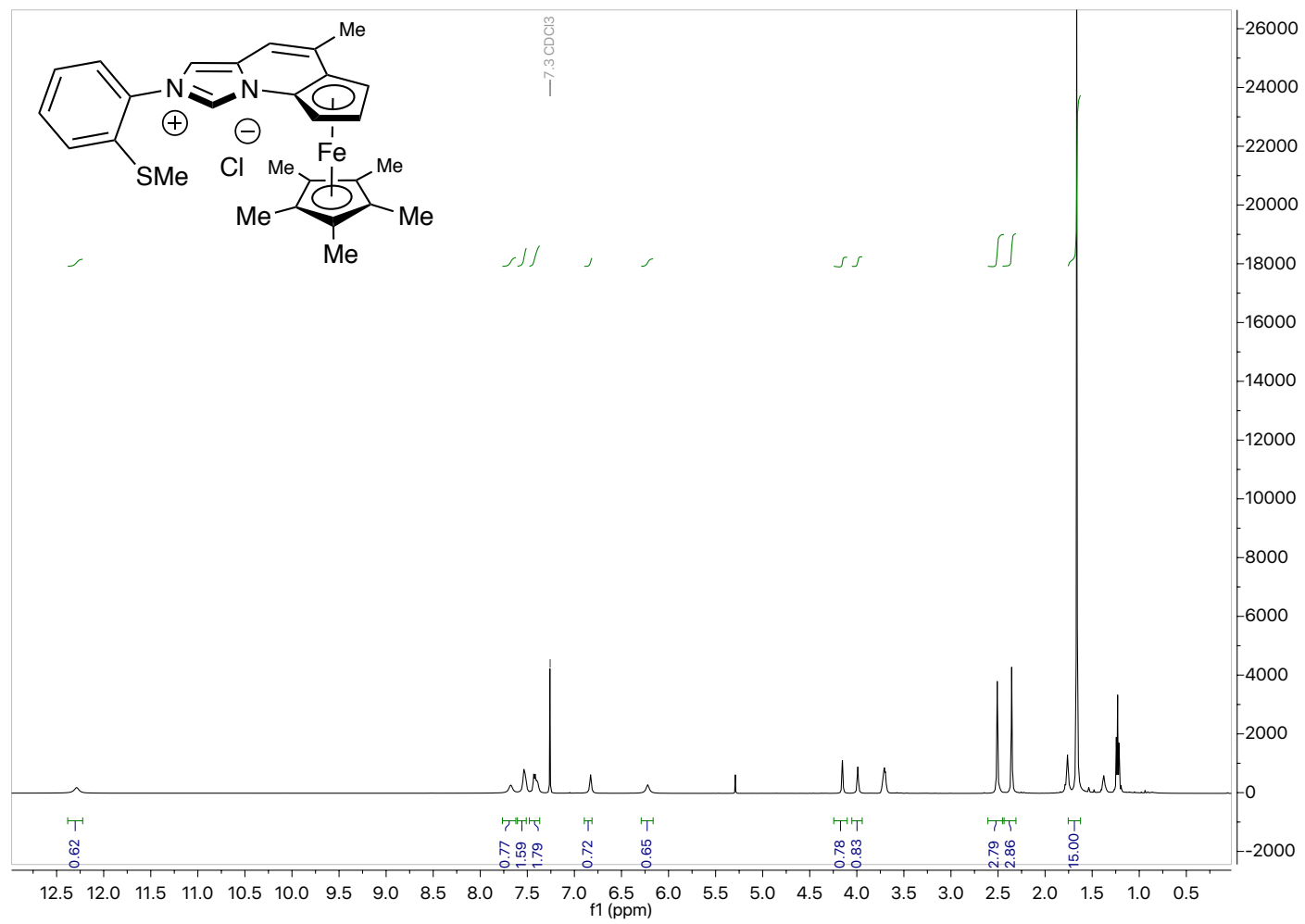

Figure S4. ${ }^{13} \mathrm{CNMR}$ of $(+)-8 \mathrm{e}$

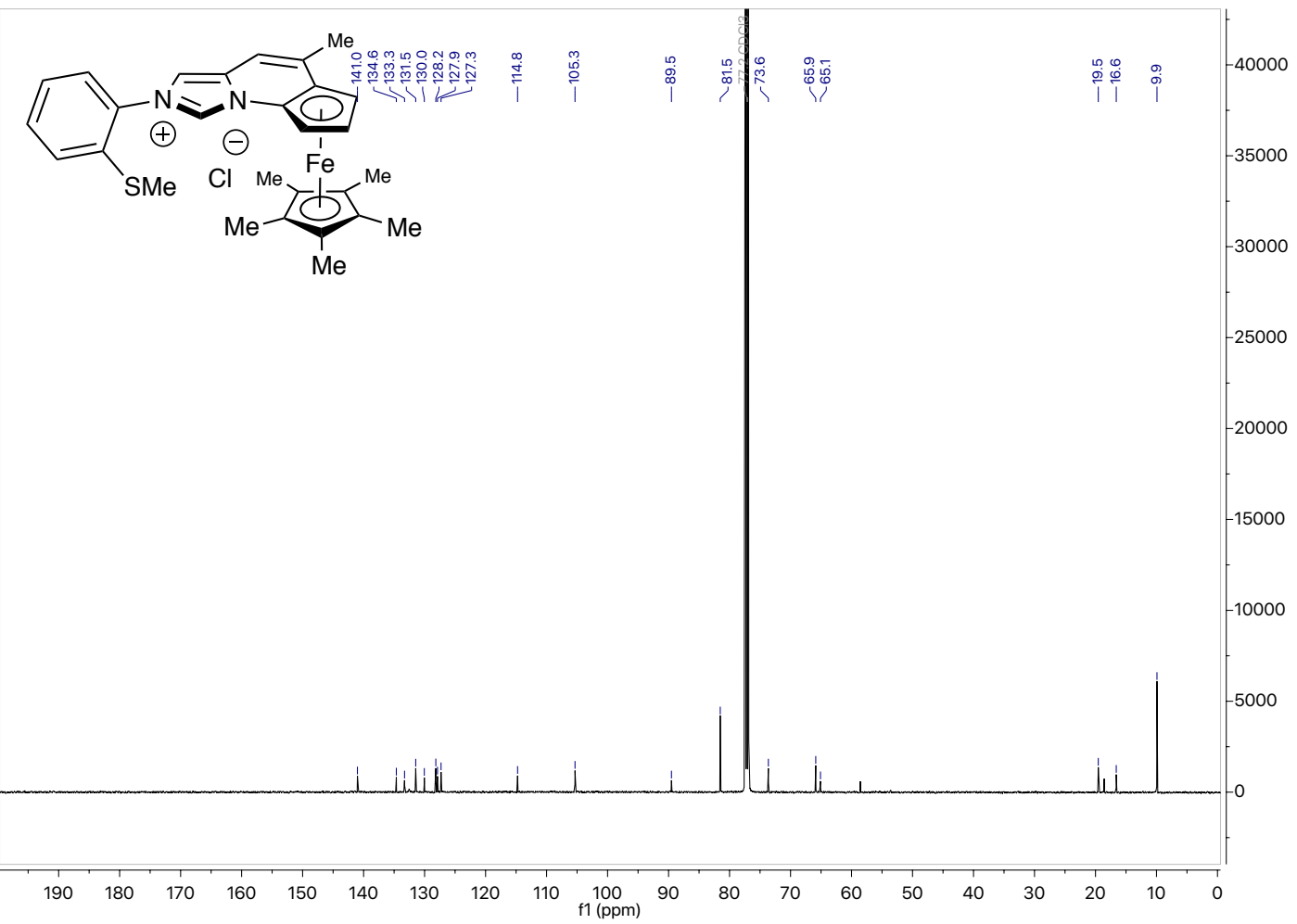


Figure S5. ${ }^{1} \mathrm{HNMR}$ of $(+)-8 \mathrm{f}$

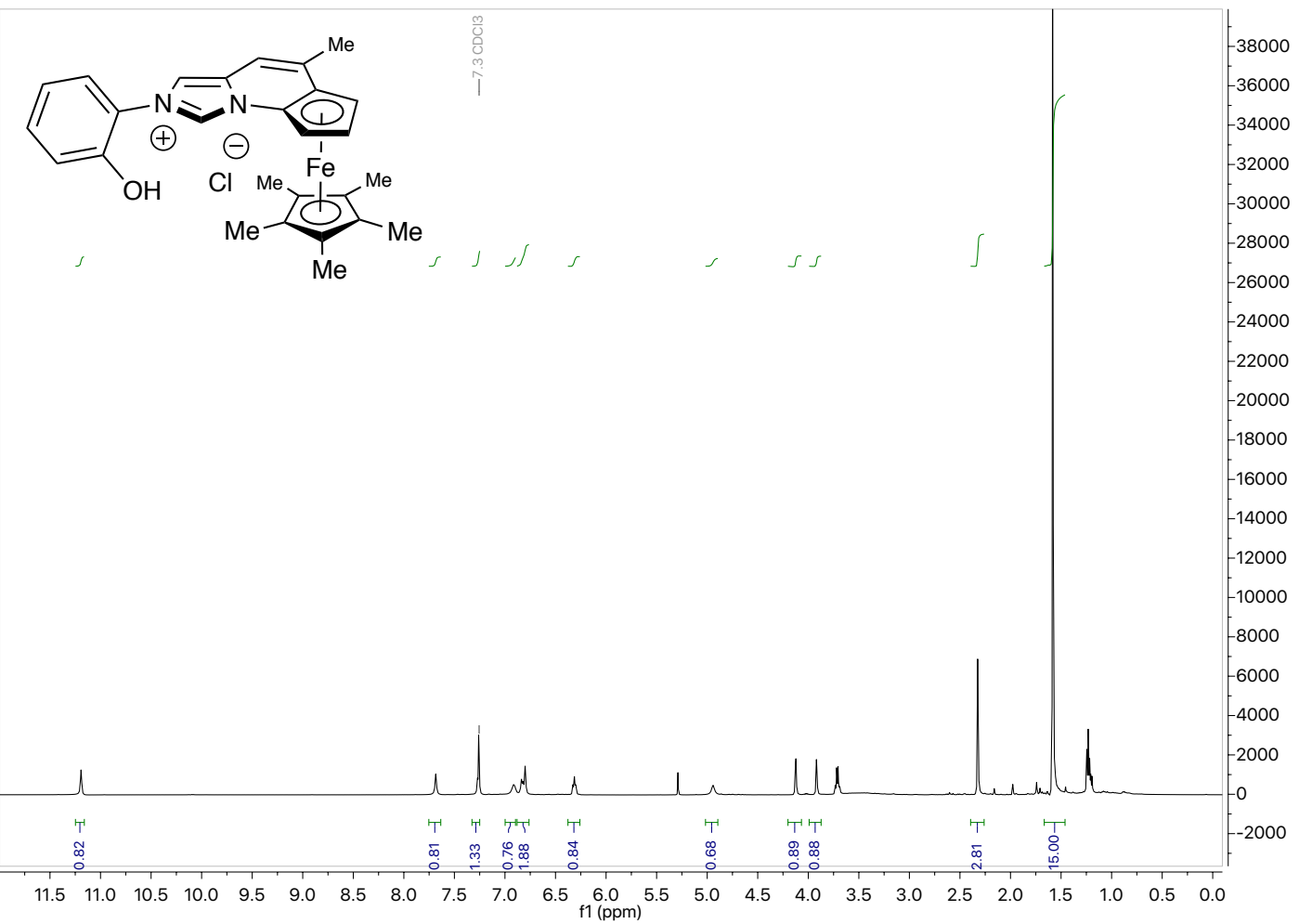

Figure S6. ${ }^{13} \mathrm{CNMR}$ of $(+)-8 \mathrm{f}$

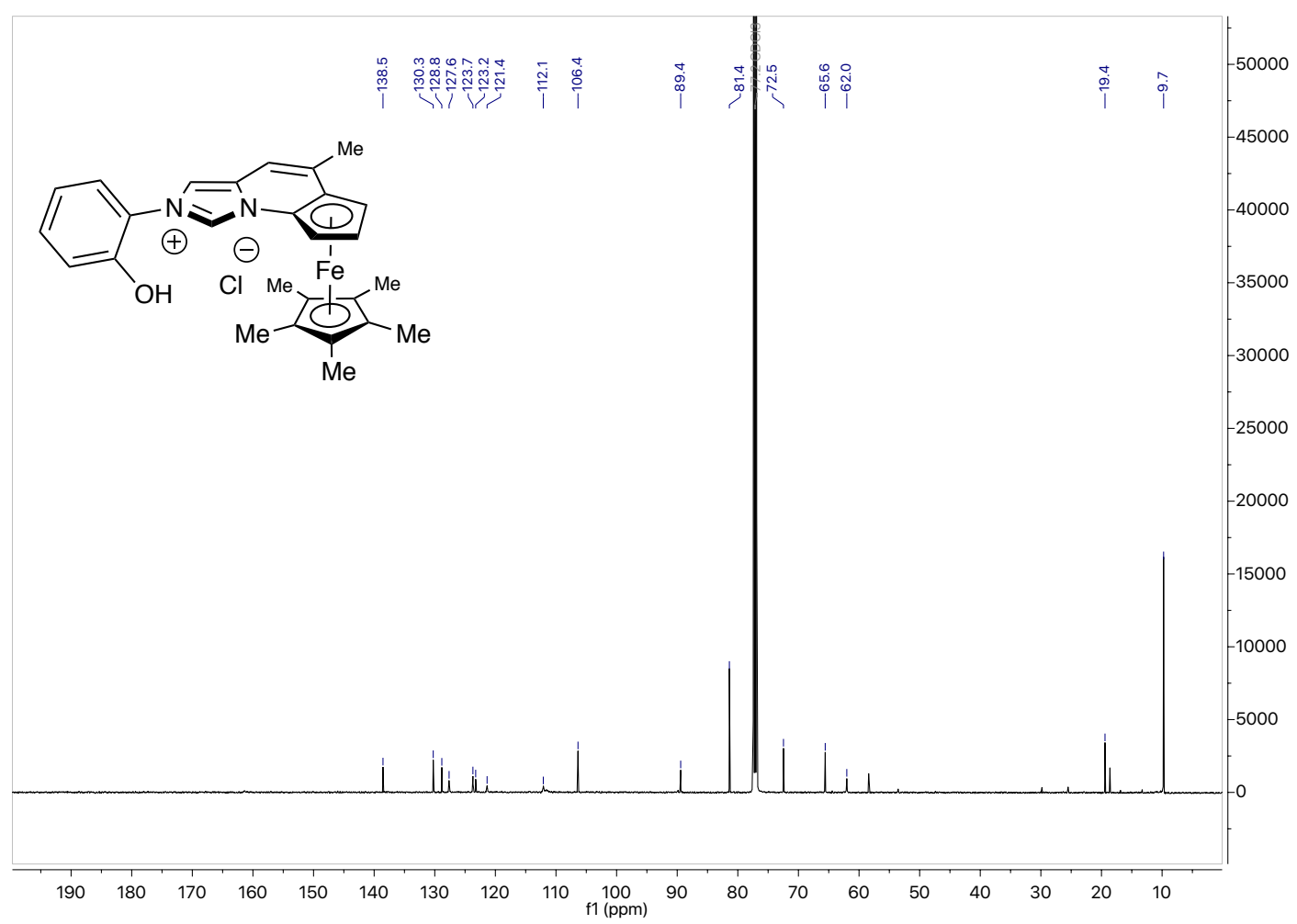


Figure S7. ${ }^{1} \mathrm{HNMR}$ of (+)-21a

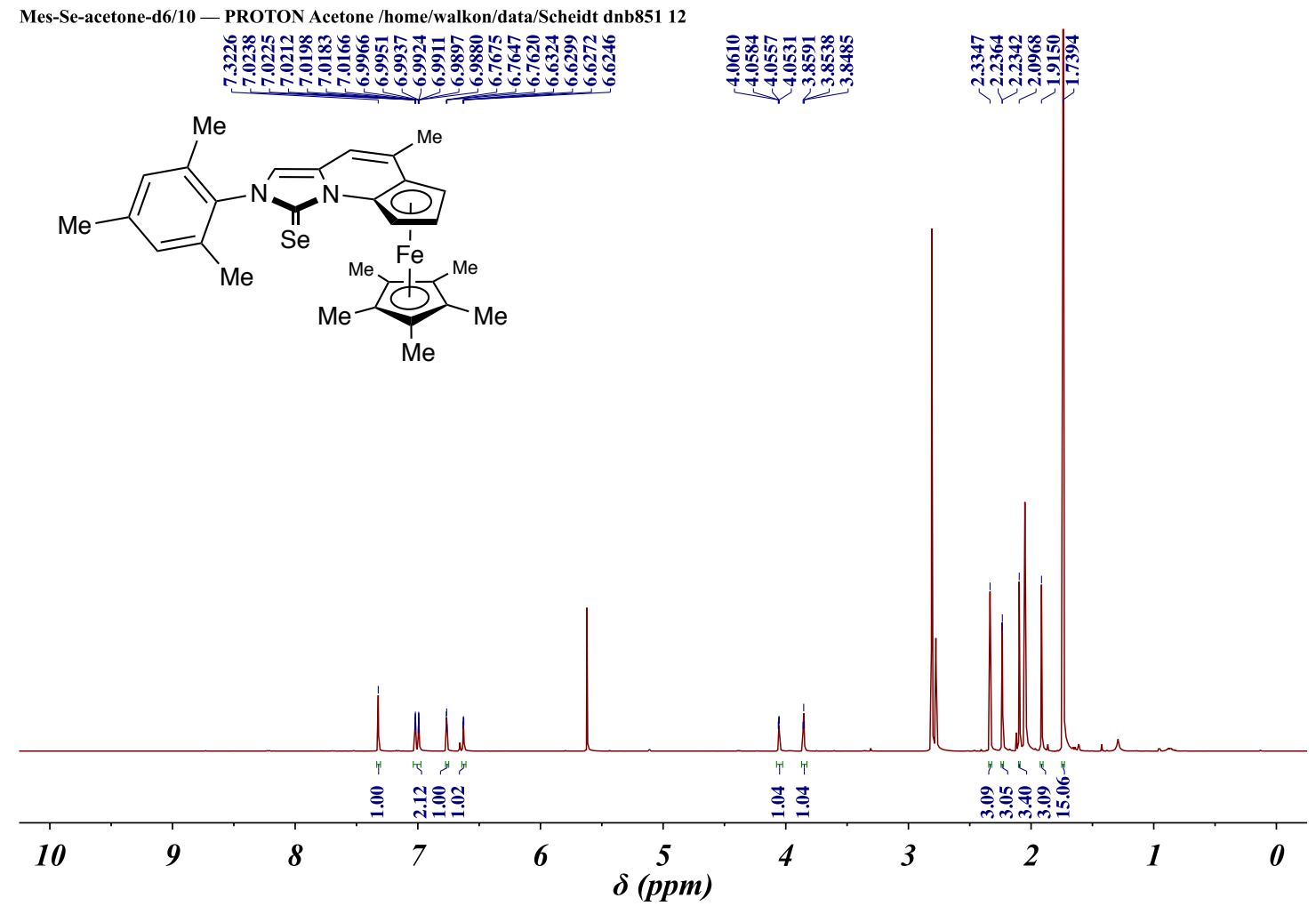

Figure S8. ${ }^{13} \mathrm{CNMR}$ of $(+)-21 \mathrm{a}$

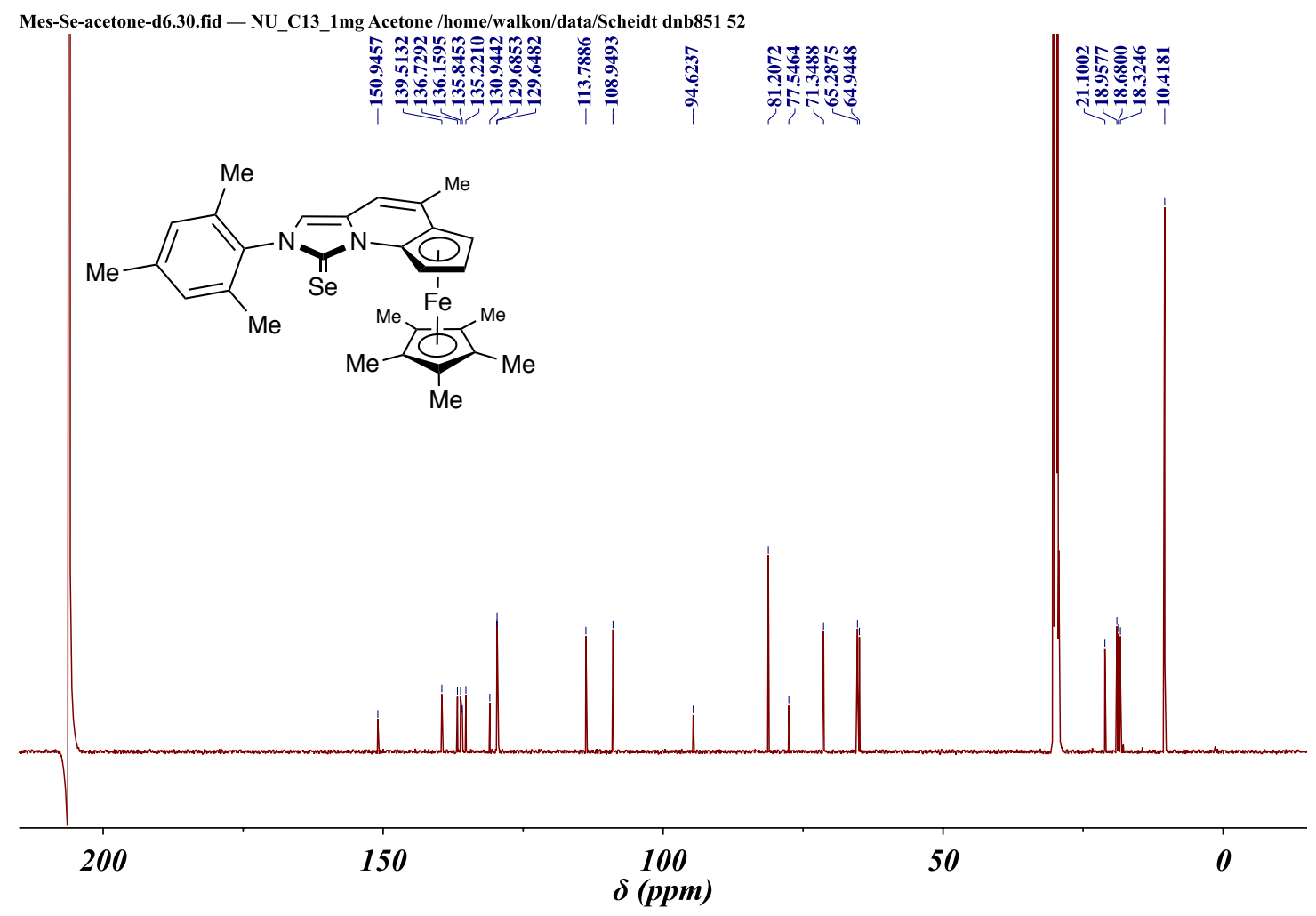


Figure S9. ${ }^{77}$ Se NMR of (+)-21a
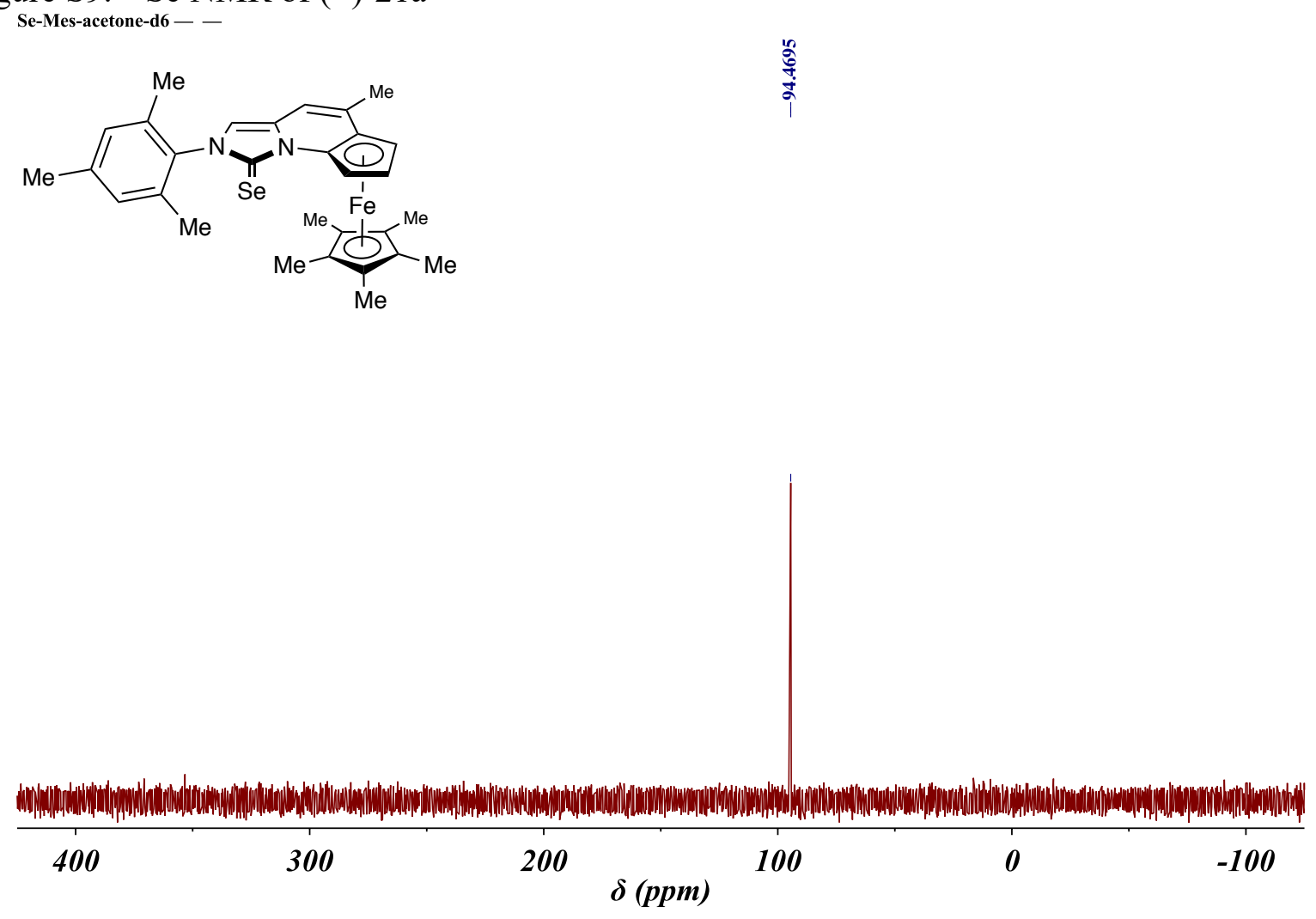
Figure S10. ${ }^{1} \mathrm{HNMR}$ of $(+)-21 \mathrm{a}$

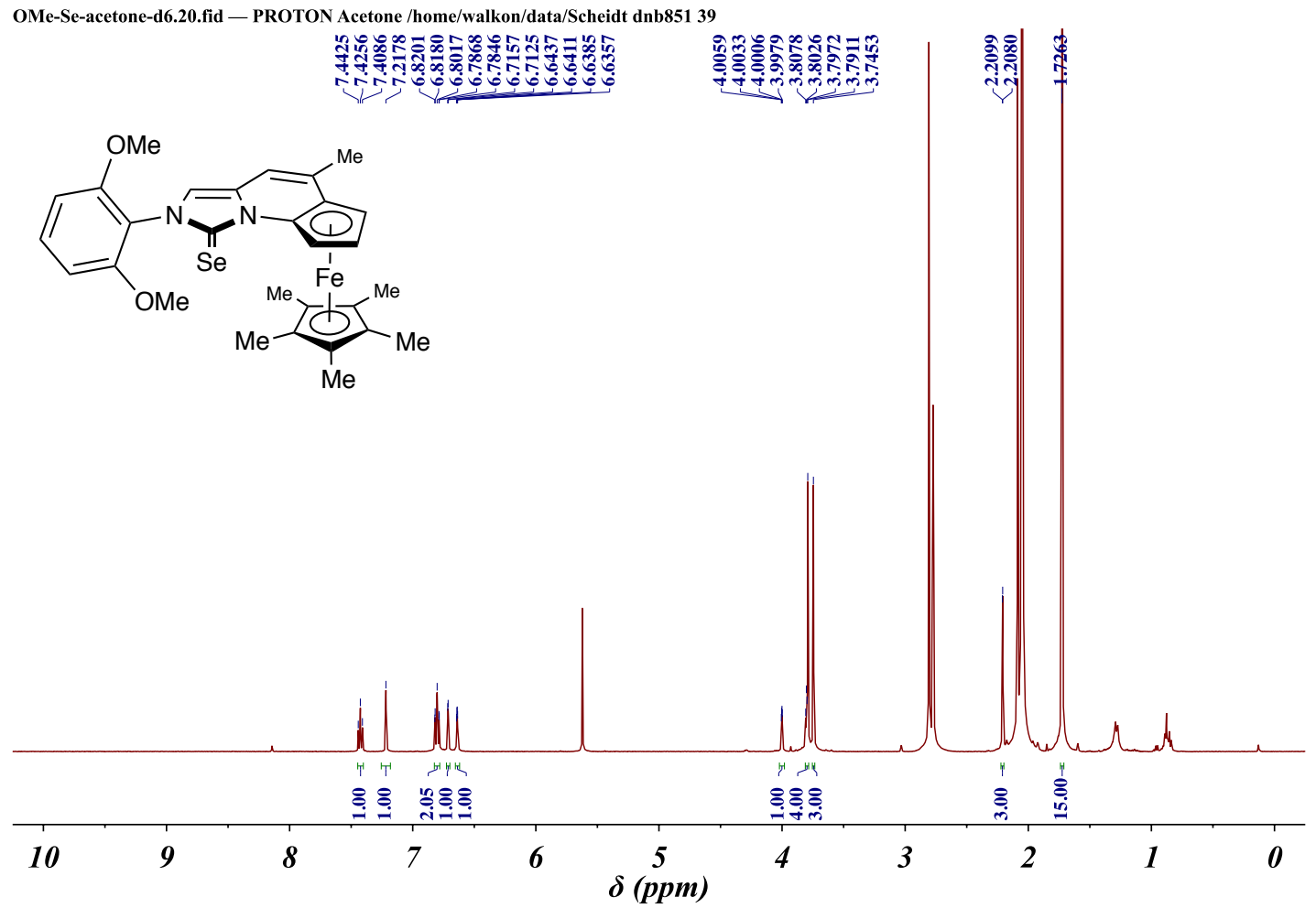

Figure S11. ${ }^{13} \mathrm{CNMR}$ of $(+)-21 \mathrm{~b}$

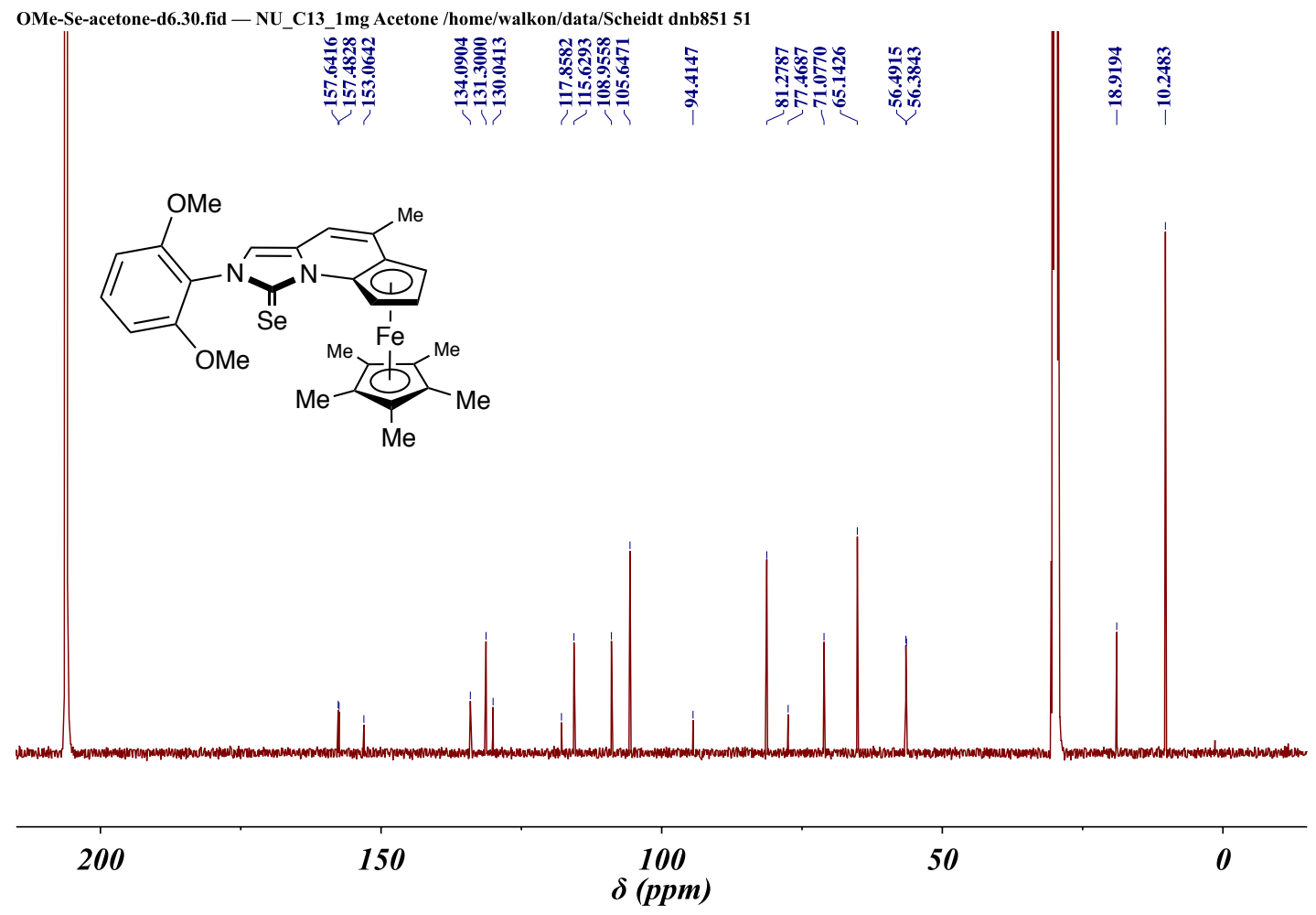


Figure S12. ${ }^{77} \mathrm{Se}$ NMR of (+)-21b
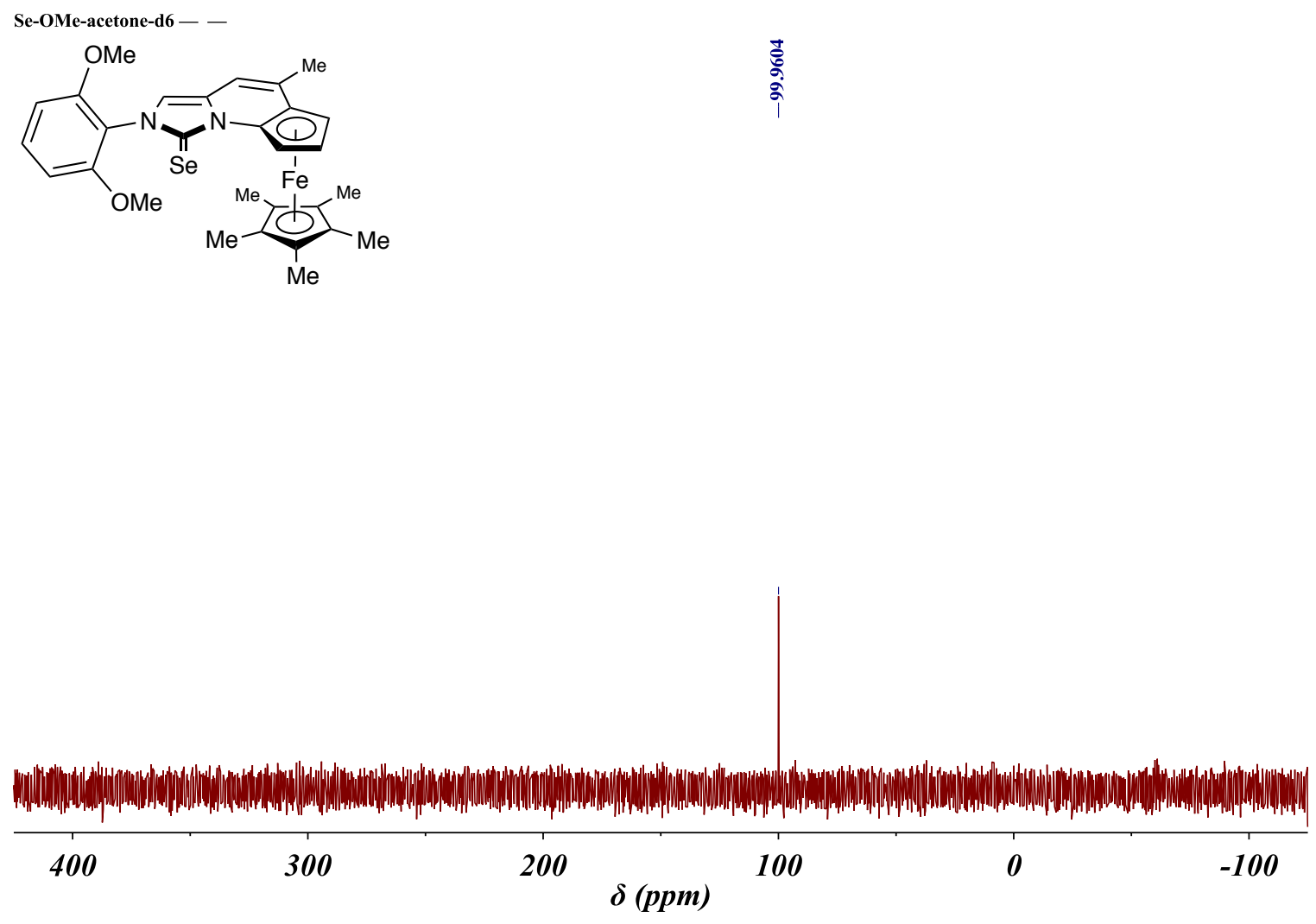
Figure S13. ${ }^{1} \mathrm{HNMR}$ of $(+)-21 \mathrm{c}$

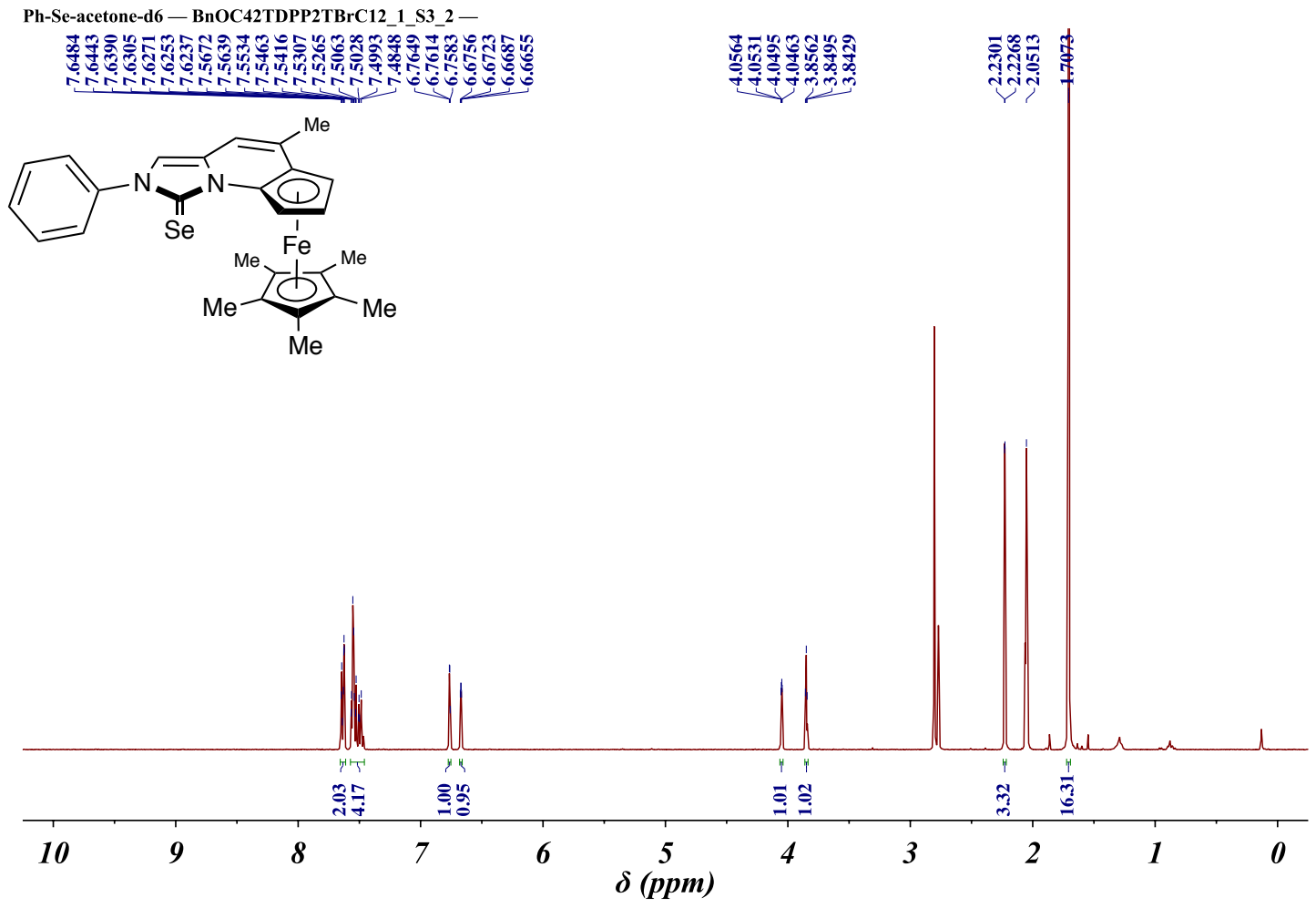

Figure S14. ${ }^{13} \mathrm{CNMR}$ of (+)-21c

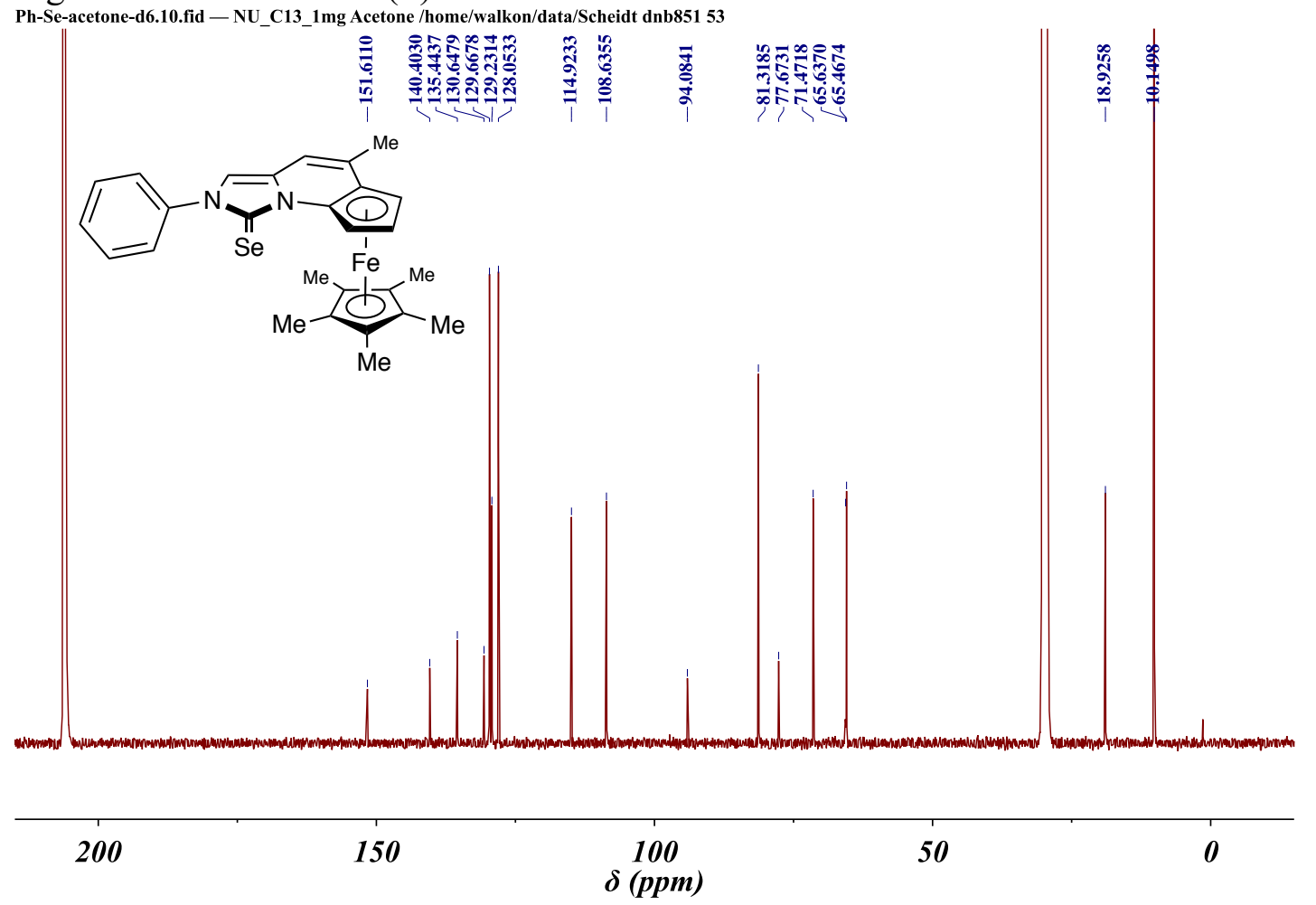


Figure S15. ${ }^{77} \mathrm{Se}$ NMR of (+)-21c
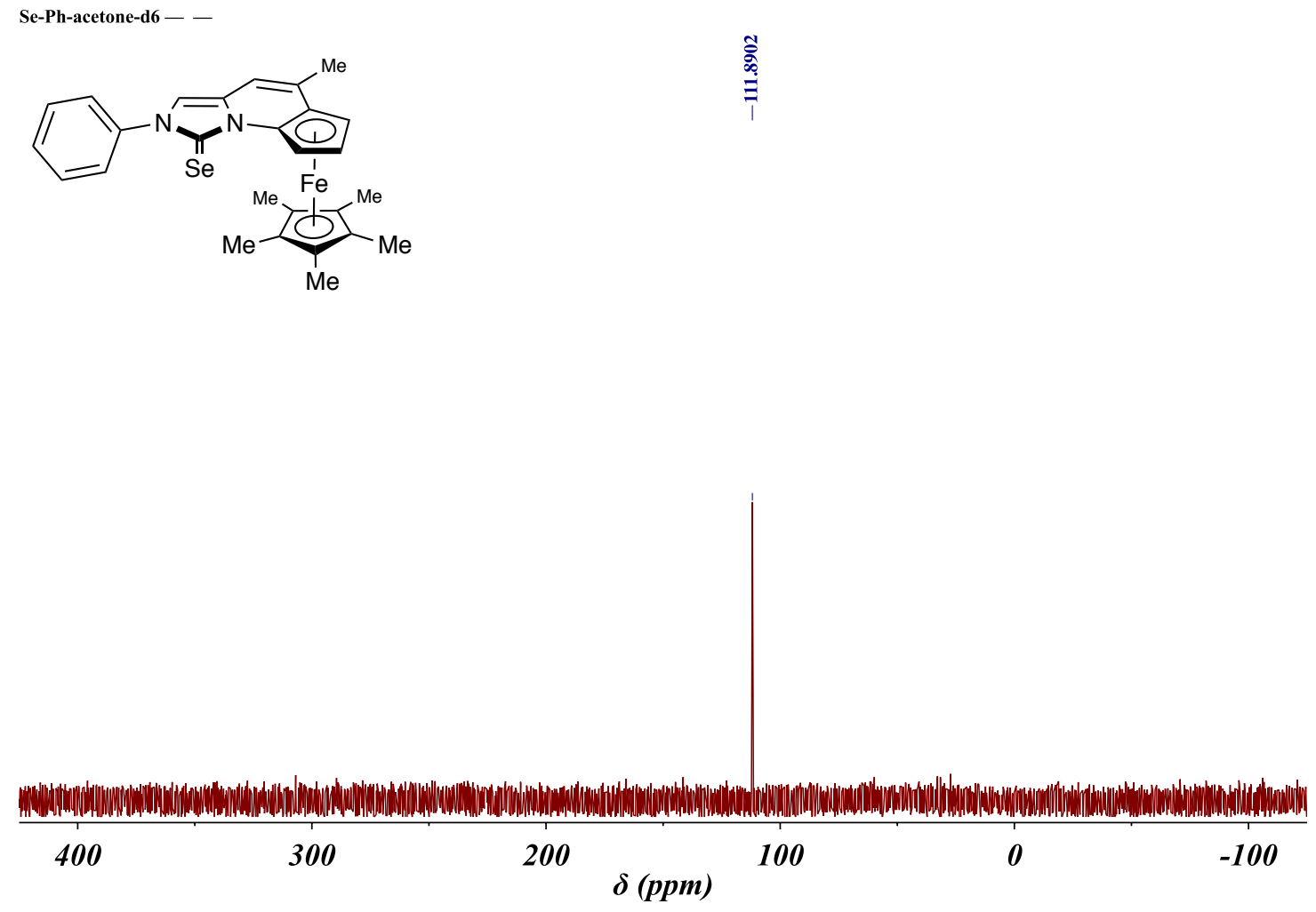
Figure S16. ${ }^{1} \mathrm{HNMR}$ of $(+)-21 \mathrm{~d}$

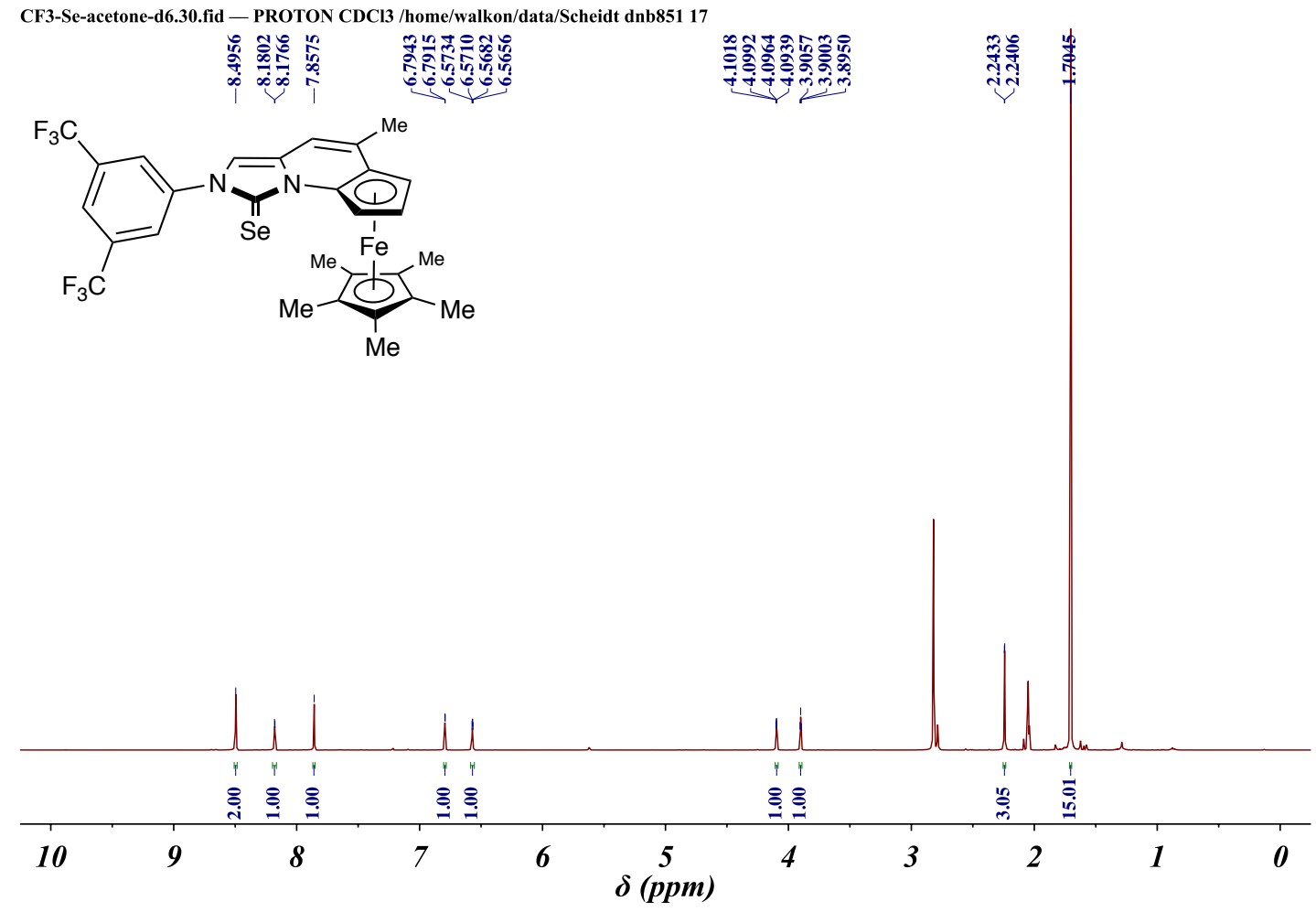

Figure S17. ${ }^{13} \mathrm{CNMR}$ of $(+)-21 \mathrm{~d}$

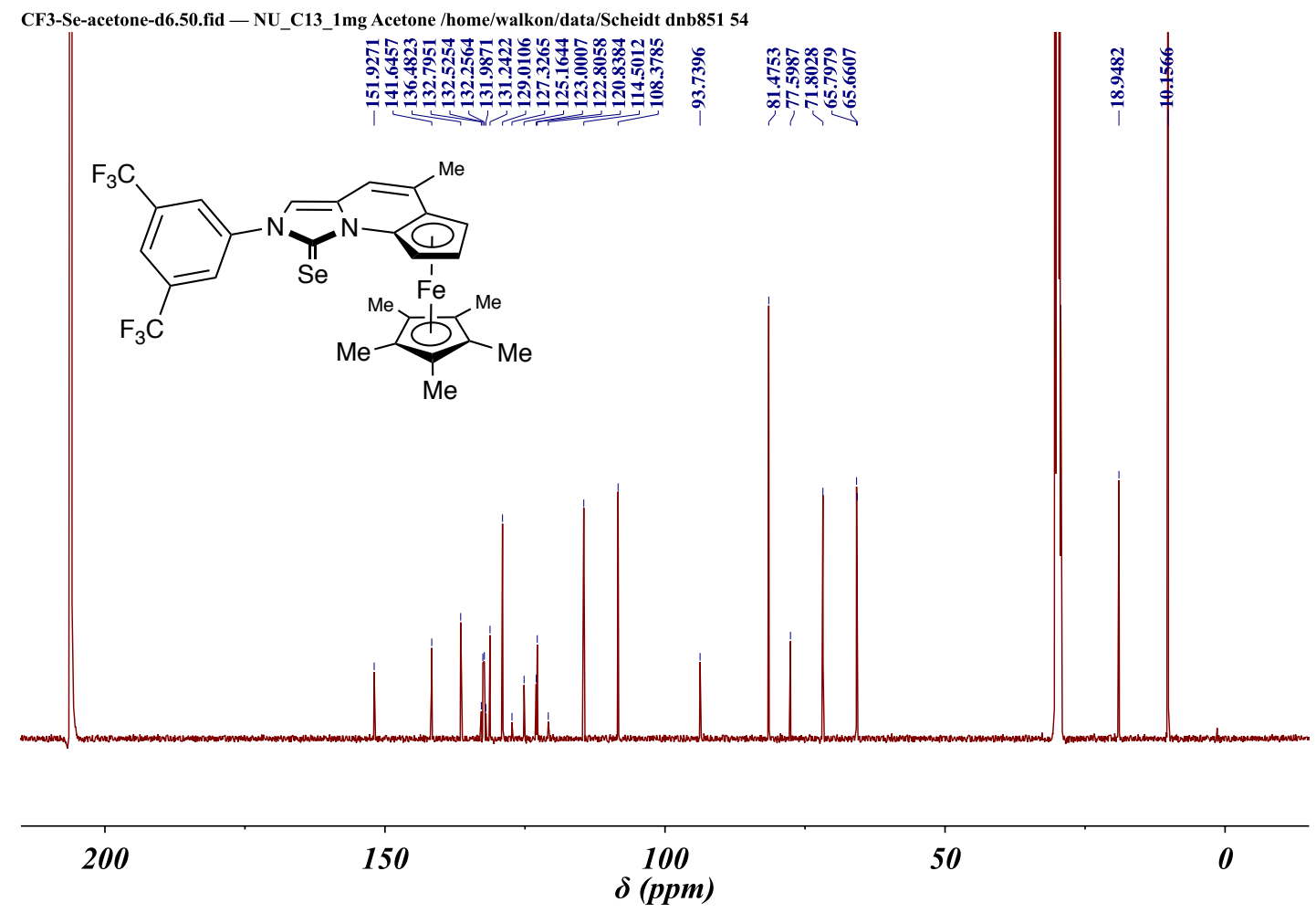


Figure S18. ${ }^{77}$ Se NMR of $(+)-21 d$
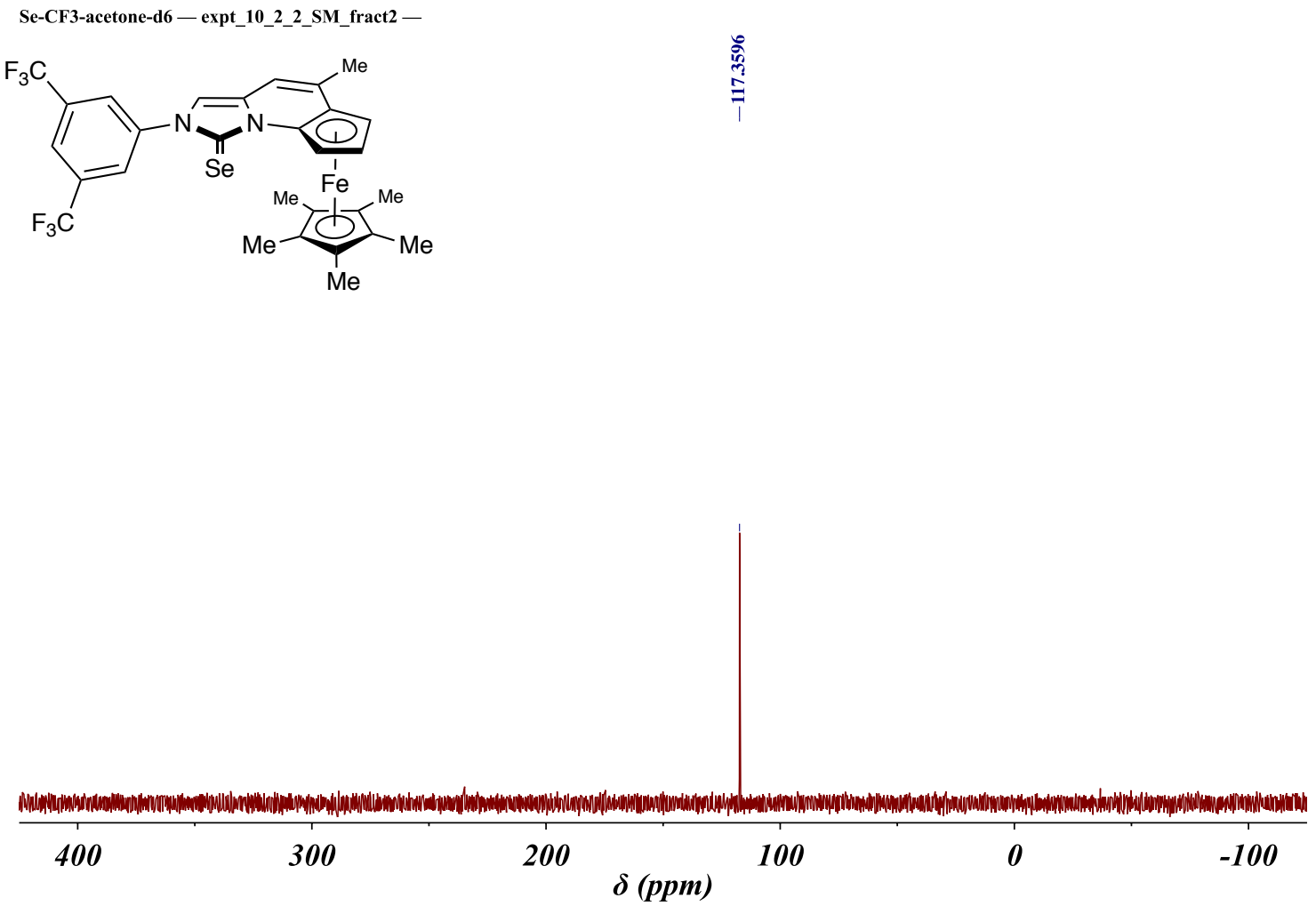


\section{References}

1. D. D. Perrin, W. L. Armarego, Purification of Laboratory Chemicals; 3rd Ed., Pergamon Press, Oxford. 1988.

2. Check, C. T.; Jang, K. P.; Schwamb, C. B.; Wong, A. S.; Wang, M. H.; Scheidt, K. A. "Ferrocene-Based Planar Chiral Imidazopyridinium Salts for Catalysis," Angew. Chem. Int. Ed. 2015, 54, 4264-4268.

3. Schwamb, C. B.; Fitzpatrick, K. P.; Brueckner, A. C.; Richardson, H. C.; Cheong, P. H. Y.; Scheidt, K. A. J. Am. Chem. Soc. 2018, 140, 10644-10648.

4. Liske, A.; Verlinden, K.; Buhl, H.; Schaper, K.; Ganter, C. Organometallics 2013, 32, 52695272 .

5. Dolomanov, O.V., Bourhis, L.J., Gildea, R.J, Howard, J.A.K. \& Puschmann, H. J. Appl. Cryst. 2009, 42, 339-341.

6. Sheldrick, G.M. Acta Cryst. 2015, A71, 3-8.

7. Sheldrick, G.M. Acta Cryst. 2015, C71, 3-8. 